\title{
Gyrokinetic linearized Landau collision operator
}

\author{
Madsen, Jens
}

Published in:

Physical Review E. Statistical, Nonlinear, and Soft Matter Physics

Publication date:

2013

Document Version

Publisher's PDF, also known as Version of record

Link back to DTU Orbit

Citation (APA):

Madsen, J. (2013). Gyrokinetic linearized Landau collision operator. Physical Review E. Statistical, Nonlinear, and Soft Matter Physics, 87(1), 011101.

\section{General rights}

Copyright and moral rights for the publications made accessible in the public portal are retained by the authors and/or other copyright owners and it is a condition of accessing publications that users recognise and abide by the legal requirements associated with these rights.

- Users may download and print one copy of any publication from the public portal for the purpose of private study or research.

- You may not further distribute the material or use it for any profit-making activity or commercial gain

- You may freely distribute the URL identifying the publication in the public portal

If you believe that this document breaches copyright please contact us providing details, and we will remove access to the work immediately and investigate your claim 


\title{
Gyrokinetic linearized Landau collision operator
}

\author{
Jens Madsen* \\ Association EURATOM-DTU, Technical University of Denmark, Department of Physics, DK-4000 Roskilde, Denmark
}

(Received 17 August 2012; published 24 January 2013)

\begin{abstract}
The full gyrokinetic electrostatic linearized Landau collision operator is calculated including the equilibrium operator, which represents the effect of collisions between gyrokinetic Maxwellian particles. First, the equilibrium operator describes energy exchange between different plasma species, which is important in multiple ion-species plasmas. Second, the equilibrium operator describes drag and diffusion of the magnetic field aligned component of the vorticity associated with the $\boldsymbol{E} \times \boldsymbol{B}$ drift. Therefore, a correct description of collisional effects in turbulent plasmas requires the equilibrium operator, even for like-particle collisions.
\end{abstract}

DOI: 10.1103/PhysRevE.87.011101

PACS number(s): $52.30 . \mathrm{Gz}, 52.20 . \mathrm{Fs}, 52.20 . \mathrm{Hv}, 52.25 . \mathrm{Xz}$

Gyrokinetic theory plays a central role in analytical and numerical investigations of low-frequency turbulence and the associated anomalous transport in magnetized laboratory plasmas and in nature [1]. The theory eliminates the fast time scale associated with the fast cyclotron gyration from the equations of motion, and reduces the dimensionality of the kinetic equation from six to five. However, gyrokinetic theory was originally developed without taking collisions into account. Collisions strongly influence low-frequency turbulence and the associated transport, they determine the steady state, and they are responsible for entropy production, ultimately making processes irreversible. Accounting for collisions in gyrokinetic theory has therefore been the subject of significant research efforts [2-7].

In a wide range of plasmas, where small-angle Coulomb collisions are dominant, collisions are well described by the Landau collision operator [8]. When the plasma is nearly in a local equilibrium state [9], the gyrokinetic distribution function can be divided into an equilibrium $F_{M a}$ part and a small perturbed $\delta F_{a}$ part. The splitting of the gyrokinetic distribution function leads to the gyrokinetic linearized collision operator describing collisions between particles of species $a$ and $b$. The operator consists of the equilibrium operator $\mathcal{C}\left[F_{M a}, F_{M b}\right]$, the test-particle operator $\mathcal{C}\left[\delta F_{a}, F_{M b}\right]$, and the field-particle operator $\mathcal{C}\left[F_{M a}, \delta F_{b}\right]$. All three operators reintroduce the fast time scale associated with the fast cyclotron gyration into the gyrokinetic equation, and are therefore explicitly gyroaveraged. The full gyroaveraged gyrokinetic test-particle operator was calculated by $\mathrm{Xu}$ and Rosenbluth [3]. In previous works the field-particle operator was often approximated [2-6] by moment restoring terms due to the intractable velocity integrals inside the field-particle operator. Only recently, Li and Ernst [7] calculated the full gyroaveraged gyrokinetic field-particle operator, which is not only more accurate, but is also less computationally expensive than previous model operators when implemented in gyrokinetic simulations. However, as in all other previous work, the equilibrium operator $\mathcal{C}\left[F_{M a}, F_{M b}\right]$ was neglected.

In this Rapid Communication we calculate the full electrostatic gyrokinetic linearized Landau collision operator including the equilibrium operator. The obtained operator is

*jmad@fysik.dtu.dk valid for arbitrary temperatures and masses, and finite Larmor radius effects are accounted for in all parts. The gyrokinetic operator is obtained by performing an explicit gyroaverage of the linearized Landau collision operator expressed in gyrocenter coordinates. The obtained equilibrium operator is responsible for energy exchange [10] between species, and therefore plays an important role in the thermalization process. The collisional energy exchange rate between species $a$ and $b$ is proportional to the ratio of the corresponding masses [11]. Therefore, the equilibrium operator is particularly important in multiple ion-species plasmas such as fusion and astrophysical plasmas. Furthermore, the equilibrium operator describes drag and diffusion of the magnetic field aligned component of the vorticity associated with the $\boldsymbol{E} \times \boldsymbol{B}$ drift. The corresponding terms have the same order of magnitude as the test- and field-particle operators. The equilibrium operator is therefore required in order to describe the influence of collisions on electrostatic turbulence and the associated transport correctly. Contrary to conventional wisdom, it is shown that the equilibrium operator must be retained even for like-particle collisions.

The starting point of our derivation is the Landau kinetic equation,

$$
\frac{\partial}{\partial t} f_{a}(z, t)+\dot{z}^{i} \frac{\partial}{\partial z^{i}} f_{a}(z, t)=\mathcal{C}\left[f_{a} ; f_{b}\right],
$$

where $f_{a}$ denotes the distribution function of species $a$ having mass $m_{a}$ and charge $q_{a}$, and $z=(\boldsymbol{x}, \boldsymbol{p})$ are noncanonical phasespace coordinates; $\boldsymbol{x}$ denotes particle position and $\boldsymbol{p}=m \boldsymbol{v}$ is the particle momentum. Repeated $i$ and $j$ imply summation throughout this Rapid Communication. The equations of motion $\dot{z}^{i}=\left\{z^{i}, h\right\}$ are here written in terms of the Hamiltonian $h$ and the Poisson bracket,

$$
\{f, g\}=\frac{\partial f}{\partial \boldsymbol{x}} \cdot \frac{\partial g}{\partial \boldsymbol{p}}-\frac{\partial g}{\partial \boldsymbol{x}} \cdot \frac{\partial f}{\partial \boldsymbol{p}}+q \boldsymbol{B} \cdot \frac{\partial f}{\partial \boldsymbol{p}} \times \frac{\partial g}{\partial \boldsymbol{p}} .
$$

The Landau collision operator,

$$
\mathcal{C}\left[f_{a} ; f_{b}\right]=\frac{\partial}{\partial p_{i}}\left[\frac{m_{a}}{m_{b}} f_{a} \frac{\partial H_{b}}{\partial p_{i}}-m_{a}^{2} \frac{\partial^{2} G_{b}}{\partial p_{i} \partial p_{j}} \frac{\partial f_{a}}{\partial p_{j}}\right],
$$

describes drag and diffusion in velocity space due to smallangle Coulomb collisions between species $a$ and $b$. The Landau collision operator in Eq. (3) is here written in terms of 
Rosenbluth potentials [12]

$$
\left(\begin{array}{c}
H_{b} \\
G_{b}
\end{array}\right)=\frac{-\Gamma_{a b}}{8 \pi} \int d^{6} z^{\prime} f_{b}\left(z^{\prime}\right) \delta^{3}\left(\boldsymbol{x}-\boldsymbol{x}^{\prime}\right)\left(\begin{array}{c}
2 u^{-1} \\
u
\end{array}\right),
$$

where $u=\left|\boldsymbol{v}-\boldsymbol{v}^{\prime}\right|$ is the relative velocity, $\Gamma_{a b}=$ $\left(q_{a} q_{b} / \epsilon_{0}\right)^{2} \ln \Lambda$, and $\delta^{3}(\boldsymbol{x})$ is the Dirac delta function. By employing the properties $\nabla_{v}^{2} G_{b}=H_{b}$ and $\nabla_{v}^{2} H_{b}=\Gamma_{a b} f_{b}$ of the Rosenbluth potentials, the collision operator Eq. (3) can be written in terms [4] of Poisson brackets:

$$
\begin{aligned}
\mathcal{C}\left[f_{a} ; f_{b}\right]= & {\left[\left\{x_{i}, f_{a}\right\}\left\{x_{i}, H_{b}\right\}\left[\frac{m_{a}}{m_{b}}-1\right]+\Gamma_{a b} \frac{m_{a}}{m_{b}} f_{a} f_{b}\right.} \\
& \left.-m_{a}^{2}\left\{x_{i},\left\{x_{j}, G_{b}\right\}\right\}\left\{x_{i},\left\{x_{j}, f_{a}\right\}\right\}\right],
\end{aligned}
$$

which simplifies the transformation of the operator to gyrocenter coordinates.

The gyrocenter coordinate transformation is a two-step transformation going from particle coordinates $z=(\boldsymbol{x}, \boldsymbol{p})$ through guiding-center coordinates $\boldsymbol{Z}_{g c}=\left(\boldsymbol{X}_{g c}, v_{\| g c}, \mu_{g c}, \theta_{g c}\right)$ to gyrocenter coordinates $\boldsymbol{Z}=\left(\boldsymbol{X}, v_{\|}, \mu, \theta\right)$. The guidingcenter coordinate transformation eliminates the fast cyclotron motion time scale in the equations of motion in the absence of fluctuating electromagnetic fields. For the sake of simplicity, nonuniformity of the magnetic field is neglected throughout. The zeroth-order particle to guiding-center coordinate transformation [13] is defined as $\boldsymbol{x}=\boldsymbol{X}_{g c}+\boldsymbol{\rho}_{0}$ and $\boldsymbol{v}=\hat{\boldsymbol{b}}\left(\boldsymbol{X}_{g c}\right) v_{\| g c}+\boldsymbol{v}_{\perp}\left(\boldsymbol{X}_{g c}, \mu_{g c}, \theta_{g c}\right)$. Here, $\boldsymbol{X}_{g c}$ is the guiding-center position, $v_{\|}$is the guiding-center velocity parallel to $\hat{\boldsymbol{b}}=\boldsymbol{B} / \boldsymbol{B}$, which denotes the unit vector parallel to the magnetic field, $\mu_{g c}$ is the magnetic dipole momentlike coordinate, and $\theta_{g c}$ denotes the gyroangle coordinate of the cyclotron motion. The perpendicular component of the velocity is related to the guiding-center coordinates as $\boldsymbol{v}_{\perp g c}=\sqrt{2 \mu_{g c} B / m} \hat{\perp}=\Omega \rho_{0} \times \hat{\boldsymbol{b}}$, where $\Omega=q B / m$ denotes the gyrofrequency, and $\hat{\perp}$ is a co-moving unit vector parallel to the perpendicular velocity vector. The unit vectors form an orthonormal triad $\hat{\boldsymbol{\theta}} \times \hat{\boldsymbol{b}}=\hat{\boldsymbol{\perp}}=\partial_{\theta_{g c}} \hat{\boldsymbol{\theta}}$, where the gyroangle is defined as $\theta_{g c}=\arctan \left[\left(\hat{\boldsymbol{e}}_{1} \cdot \boldsymbol{v}_{\perp}\right) /\left(\hat{\boldsymbol{e}}_{2} \cdot \boldsymbol{v}_{\perp}\right)\right]$ and $\hat{\boldsymbol{e}}_{1}$ and $\hat{\boldsymbol{e}}_{2}$ are local fixed orthonormal basis vectors, which, together with $\hat{\boldsymbol{b}}$, form a local orthonormal triad. The fixed unit vectors $\hat{\boldsymbol{e}}_{1}$ and $\hat{\boldsymbol{e}}_{2}$ are related to the co-moving unit vectors through the relation $\hat{\perp}\left(\boldsymbol{X}_{g c}, \theta_{g c}, t\right)=-\hat{\boldsymbol{e}}_{1}\left(\boldsymbol{X}_{g c}, t\right) \sin \theta_{g c}-\hat{\boldsymbol{e}}_{2}\left(\boldsymbol{X}_{g c}, t\right) \cos \theta_{g c}$.

In the second step a fluctuating small-amplitude electric field is introduced. The fluctuating electric field reintroduces the fast time scale associated with the cyclotron motion into the guiding-center equations of motion. The fast time scale can be asymptotically eliminated by adopting the standard nonlinear gyrokinetic ordering, $q \phi / T \sim \omega / \Omega \sim k_{\|} / k_{\perp} \sim \epsilon_{\delta} \ll 1$ and $k_{\perp} \rho_{i} \sim 1$, where $\phi$ denotes the electrostatic potential, $T$ is the temperature, $\omega$ is the characteristic fluctuation frequency, and $k_{\|}$and $k_{\perp}$ are the characteristic parallel and perpendicular fluctuation wave numbers, respectively. The resulting guidingcenter to gyrocenter coordinate transformation [1] is given as

$$
\boldsymbol{Z}=\boldsymbol{Z}_{g c}+\epsilon_{\delta}\left\{S_{1}, \boldsymbol{Z}_{g c}\right\}+O\left(\epsilon_{\delta}^{2}\right) .
$$

Here, $S_{1}=\Omega^{-1} \int_{0}^{\theta} d \theta^{\prime} q \tilde{\phi}$, is the first-order generating function of the Lie transformation and $\tilde{\phi}=\phi-\langle\phi\rangle$ defines the oscillatory part of the electrostatic potential; $\langle\phi\rangle=(2 \pi)^{-1} \int_{0}^{2 \pi} d \theta \phi$ is the gyroangle average. To first order in $\epsilon$, the Poisson bracket written in terms of gyrocenter coordinates [14] of two arbitrary functions $F$ and $G$ is given as

$$
\begin{aligned}
\{F, G\}= & \frac{q}{m}\left(\frac{\partial F}{\partial \theta} \frac{\partial G}{\partial \mu}-\frac{\partial F}{\partial \mu} \frac{\partial G}{\partial \theta}\right) \\
& +\frac{\hat{\boldsymbol{b}}}{m} \cdot\left(\nabla F \frac{\partial G}{\partial u}-\nabla G \frac{\partial F}{\partial u}\right)-\frac{\hat{\boldsymbol{b}}}{q B} \cdot \nabla F \times \nabla G .
\end{aligned}
$$

With the gyrokinetic Poisson bracket (7) and the gyrocenter coordinate transformation (6) at hand, the gyroaveraged kinetic equation (1), written in terms of gyrocenter coordinates, becomes

$$
\frac{\partial}{\partial t}\left\langle F_{a}\right\rangle+\dot{Z}_{i} \frac{\partial}{\partial Z_{i}}\left\langle F_{a}\right\rangle=\sum_{a, b}\left\langle\mathcal{C}\left[\left\langle F_{a}\right\rangle ;\left\langle F_{b}\right\rangle\right]\right\rangle,
$$

where $F_{a}(\boldsymbol{Z}, t) \doteq f_{a}(z, t)$ is the gyrokinetic distribution function, $\dot{Z}_{i}=\left\{Z_{i}, \mathcal{H}_{a}\right\}$ is the gyroangle-independent gyrokinetic equations of motion, and $\mathcal{H}_{a}$ is the gyrokinetic Hamiltonian. When collisional effects are neglected, it can be shown [14] that the oscillatory part $\tilde{F}_{a}=F_{a}-\left\langle F_{a}\right\rangle$ vanishes, and hence an equation for $\left\langle F_{a}\right\rangle$ is readily obtained. However, when collisions are accounted for, $\left\langle F_{a}\right\rangle$ and $\tilde{F}_{a}$ do not readily decouple, but it can be shown that the approximation $\left\langle C\left[F_{a} ; F_{b}\right]\right\rangle \simeq$ $\left\langle C\left[\left\langle F_{a}\right\rangle ;\left\langle F_{b}\right\rangle\right]\right\rangle$ suffices [4] in strongly magnetized plasmas with $v / \Omega \sim \epsilon_{v} \ll 1 ; v$ is a characteristic collision frequency.

The gyrokinetic linearized Landau collision operator enters the widely applied $\delta F$ gyrokinetic equation [1], which governs the time evolution of the gyroangle-independent perturbed distribution function $\delta F_{a}$. The equilibrium distribution function is taken as the lowest-order stationary solution to the zeroth-order gyroaveraged kinetic equation (8), namely, a gyrokinetic Maxwellian $F_{M a}=N\left(2 \pi T m_{a}\right)^{-3 / 2}$ $\exp \left(-\frac{1 / 2 m_{a} v_{\|}^{2}+\mu B}{T}\right)$, where $N$ and $T$ are the equilibrium gyrocenter density and temperature, respectively. The amplitude of $\delta F_{A}$ is assumed small compared to the equilibrium distribution function $\delta F_{a} / F_{M a} \sim \epsilon_{\delta}$. In the following calculations, no distinction will be made between the gyrokinetic expansion parameter and the degree of magnetization $\epsilon \sim \epsilon_{\delta} \sim \epsilon_{\nu}$.

The $\delta F$ gyrokinetic equation is formally of order $\epsilon^{2}$. In the calculations of the gyroaveraged collision operator, terms up to second order must therefore be retained. The test-particle $\mathcal{C}\left[\delta F_{a}, F_{M b}\right]$ and the field-particle $\mathcal{C}\left[F_{M a}, \delta F_{b}\right]$ operators are of order $\epsilon^{2}$, whereas the equilibrium collision operator $\mathcal{C}\left[F_{M a}, F_{M b}\right]$ is formally of order $\epsilon$, and hence firstand second-order contributions must be retained.

When the gyrokinetic linearized collision operator is included in the modern gyrokinetic theory, where the polarization density is treated explicitly [15], it is implicitly assumed, e.g., Refs. [16-20], that the equilibrium part vanishes in likeparticle collisions. This conventional wisdom is most probably emerged because the Landau collision operator vanishes, $\mathcal{C}\left[f_{a M}, f_{b M}\right]=0$, when evaluated using two Maxwellians $f_{M}=n_{0}\left(2 \pi m T_{0}\right)^{-3 / 2} \exp \left(\frac{-m v^{2}}{2 T_{0}}\right)$ with the same temperature $T_{0} ; n_{0}$ is the equilibrium particle density. However, the Landau collision operator does not vanish [3] when operating on two gyrokinetic Maxwellians $F_{M}$. Using the gyrocenter coordinate 
transformation, given in Eq. (6), the gyrokinetic Maxwellian can be written as $F_{M}(\boldsymbol{Z})=f_{M}(\boldsymbol{z})[1-\epsilon q \tilde{\phi}(\boldsymbol{z}) / T]+O\left(\epsilon^{2}\right)$, showing that $F_{M}$ and $f_{M}$ are not identical, and hence $\mathcal{C}\left[F_{M a}, F_{M a}\right]$ contains a second-order contribution which must be retained.

We note that $F_{M a}(\boldsymbol{Z})(1-q\langle\phi\rangle(\boldsymbol{Z}) / T)=f_{M a}(\boldsymbol{z})$ $[1-q \phi(\boldsymbol{x}) / T]+O\left(\epsilon^{2}\right)$, and hence the like-particle gyrokinetic equilibrium collision operator can be written as

$$
\mathcal{C}\left[F_{M a}, F_{M a}\right]=\mathcal{C}\left[F_{M a} \frac{q\langle\phi\rangle}{T}, F_{M a}\right]+\mathcal{C}\left[F_{M a}, F_{M a} \frac{q\langle\phi\rangle}{T}\right],
$$

which can be evaluated using the gyrokinetic test-particle operator Eq. (15) and the field-particle operator Eq. (20) operator given later, since the arguments $F_{M a} \frac{q\langle\phi\rangle}{T}$ are gyroangle independent.

For unlike-particle collisions we must explicitly calculate the gyroaverage of the gyrokinetic equilibrium collision operator $\mathcal{C}\left[F_{M a}, F_{M b}\right]$ retaining all second-order terms. The secondorder contributions originate from the velocity derivatives and the Rosenbluth potentials when expressed in gyrocenter coordinates. The velocity derivatives $\frac{\partial}{\partial v}=m\{\boldsymbol{x}, \cdot\}$ give rise to second-order contributions because the particle position $\boldsymbol{x}=$ $\boldsymbol{X}+\boldsymbol{\rho}_{0}+\epsilon \boldsymbol{\rho}_{1}$ includes the first-order gyrocenter displacement $\rho_{1}=-\left\{S_{1}, \boldsymbol{X}+\boldsymbol{\rho}_{0}\right\}$. When expressed in gyrocenter coordinates the relative velocity in the Rosenbluth potentials Eq. (4) becomes

$$
u=\left[1-\left\{S_{1}, Z_{i}\right\} \partial_{Z_{i}}-\left\{S_{1}^{\prime}, Z_{i}^{\prime}\right\}^{\prime} \partial_{Z_{i}^{\prime}}\right] u_{0},
$$

where $S_{1}^{\prime}$ and $\{\cdot, \cdot\}^{\prime}$ denote the first-order generating function and the Poisson bracket expressed in primed coordinates, respectively; $v_{0}=\hat{\boldsymbol{b}} v_{\|}+\boldsymbol{v}_{\perp}(\boldsymbol{Z})$ denotes the zeroth-order velocity and $u_{0}=\left|\boldsymbol{v}_{0}-\boldsymbol{v}_{0}^{\prime}\right|$. Inserting the transformed relative velocity (10) into the Rosenbluth potentials, we get

$$
\left(\begin{array}{c}
H_{b}\left[F_{M b}\right] \\
G_{b}\left[F_{M b}\right]
\end{array}\right)=\left(\begin{array}{c}
H_{b 0} \\
G_{b 0}
\end{array}\right)-\frac{q_{a} \tilde{\phi}}{m_{a} v_{0}}\left(\begin{array}{c}
H_{b 0}^{\prime} \\
G_{b 0}^{\prime}
\end{array}\right)-\left(\begin{array}{c}
h^{\phi_{b}} \\
g^{\phi_{b}}
\end{array}\right),
$$

where $H_{b 0}$ and $G_{b 0}$ are the Rosenbluth potentials evaluated with the zeroth-order relative velocity $\left|\boldsymbol{v}_{0}-\boldsymbol{v}_{0}^{\prime}\right|, G_{0 b}^{\prime}=$ $d G_{0 b} / d v$, and

$$
\begin{aligned}
\left(\begin{array}{c}
h^{\phi_{b}} \\
g^{\phi_{b}}
\end{array}\right)= & -\frac{\Gamma_{a b}}{8 \pi} \int d^{6} Z^{\prime} F_{M} \delta\left(\boldsymbol{x}^{\prime}-\boldsymbol{x}\right) \\
& \times\left\{S_{1}^{\prime},\left(\begin{array}{c}
2\left|\boldsymbol{v}_{0}-\boldsymbol{v}_{0}^{\prime}\right|^{-1} \\
\left|\boldsymbol{v}_{0}-\boldsymbol{v}_{0}^{\prime}\right|
\end{array}\right)\right\}
\end{aligned}
$$

are the field-particle-like parts that originate from the coordinate transformation of $\boldsymbol{v}^{\prime}$ entering the relative velocity (10). The two first terms in $H_{b}\left[F_{M b}\right]$ and in $G_{b}\left[F_{M b}\right]$, Eq. (11), are time independent because they are evaluated with $F_{M b}$. Therefore, the calculations of these terms resemble those of the test-particle operator $\mathcal{C}\left[\delta F_{a}, F_{M b}\right]$. It is therefore helpful to divide the equilibrium collision operator into test-particle-like $\mathcal{C}_{M}^{T}$ and field-particle-like $\mathcal{C}_{M}^{F}$ parts,

$$
\mathcal{C}\left[F_{M a}, F_{M b}\right]=\mathcal{C}_{M}^{T}+\mathcal{C}_{M}^{F},
$$

where $\mathcal{C}_{M}^{F}$ contains all terms in $\mathcal{C}\left[F_{M a}, F_{M b}\right]$ that are evaluated with the field-particle-like Rosenbluth potentials $h^{\phi_{b}}$ and $g^{\phi_{b}}$, Eq. (12). All remaining terms in $\mathcal{C}\left[F_{M a}, F_{M b}\right]$ are described by $\mathcal{C}_{M}^{T}$.

In the explicit calculations of the gyroaverage of $\mathcal{C}_{M}^{T}$ the electrostatic potential is written in terms of its inverse Fourier transform $\phi(x)=(2 \pi)^{-3 / 2} \int d^{3} k e^{i k \cdot x} \phi_{k}$, and the Bessel function identity $e^{i k_{\perp} \rho_{0} \cos \theta}=J_{0}\left(k_{\perp} \rho_{0}\right)+2 \sum_{l=1}^{\infty} J_{l}\left(k_{\perp} \rho_{0}\right) i^{l} \cos l \theta$ is used; $J_{n}$ denotes the $n$ th-order Bessel function of the first kind. Using these definitions the gyroaverage of $\mathcal{C}_{M}^{T}$ becomes

$$
\begin{aligned}
\left\langle\mathcal{C}_{M}^{T}\right\rangle= & \int d^{3} k e^{i \boldsymbol{k} \cdot X}\left\{\left(1-\frac{m_{a}}{m_{b}}\right) v_{s} F_{a M}\left[\frac{m_{a} v_{0}^{2}}{T_{a}}+\frac{q_{a} \rho_{0}^{2} k_{\perp}^{2} \phi_{k}}{2 T_{a}}\left[J_{0}\left(k_{\perp} \rho_{0}\right)+J_{2}\left(k_{\perp} \rho_{0}\right)\right]\right]+\Gamma_{a b} \frac{m_{a}}{m_{b}} F_{M a} F_{M b}\right. \\
& +v_{D} F_{a M}\left[-\frac{m_{a} v_{0}^{2}}{T_{a}}-\frac{q_{a} \rho_{0}^{2} k_{\perp}^{2} \phi_{k}}{2 T_{a}}\left\{J_{0}\left(k_{\perp} \rho_{0}\right)\left[1+\frac{2 v_{0}^{2}}{c_{\perp}^{2}}\right]+\frac{J_{2}\left(k_{\perp} \rho_{0}\right)}{2}\left[1-\frac{m_{a} v_{0}^{2}}{T_{a}}\right]\right\}\right] \\
& \left.+v_{\|} F_{a M}\left[\frac{m_{a} v_{0}^{2}}{2 T_{a}}\left[\frac{m_{a} v_{0}^{2}}{T_{a}}-1\right]-\frac{q_{a} \rho_{0}^{2} k_{\perp}^{2} \phi_{k}}{2 T_{a}}\left(J_{0}\left(k_{\perp} \rho_{0}\right)\left[1-\frac{m v_{0}^{2}}{T_{a}}\right]-\frac{J_{2}\left(k_{\perp} \rho_{0}\right)}{2}\left[1+\frac{2 m_{a} v_{0}^{2}}{T_{a}}\right]\right)\right]\right\},
\end{aligned}
$$

where $v_{0}^{2}=v_{\|}^{2}+2 \mu B / m_{a}$, and $v_{s}=\left(m_{a}^{2} v_{0}\right)^{-1} d H_{0 b} / d v_{0}, \quad v_{D}=-2\left(m_{a}^{2} v_{0}^{3}\right)^{-1} d G_{0 b} / d v_{0}$, and $v_{\|}=-2\left(m_{a}^{2} v_{0}^{2}\right)^{-1} d^{2} G_{0 b} / d v_{0}^{2}$ denote the standard slowing-down, deflection, and parallel velocity diffusion frequencies [21], respectively. For completeness we also give the gyroaverage of the test-particle collision operator [3]

$$
\begin{aligned}
\left\langle\mathcal{C}\left[\delta F_{a}, F_{M b}\right]\right\rangle= & v_{s}\left(\frac{m_{a}}{m_{b}}-1\right)\left[2 \mu \frac{\partial}{\partial \mu}+v_{\|} \frac{\partial}{\partial v_{\|}}\right] \delta F_{a}+\Gamma_{a b} \frac{m_{a}}{m_{b}} \delta F_{a} F_{M b} \\
& +v_{D}\left[\frac{\mu B+m v_{\|}^{2}}{2 m_{a} \Omega_{a}^{2}} \nabla_{\perp}^{2}+\frac{\mu B+m v_{\|}^{2}}{B} \frac{\partial}{\partial \mu}+\frac{\mu m v_{\|}^{2}}{B} \frac{\partial^{2}}{\partial \mu^{2}}-2 \mu v_{\|} \frac{\partial^{2}}{\partial \mu \partial v_{\|}}+\frac{\mu B}{m_{a}} \frac{\partial^{2}}{\partial v_{\|}^{2}}\right] \delta F_{a} \\
& +v_{\|}\left[\frac{\mu B}{2 m_{a} \Omega_{a}^{2}} \nabla_{\perp}^{2}+\mu \frac{\partial}{\partial \mu}+2 \mu^{2} \frac{\partial^{2}}{\partial \mu^{2}}+2 \mu v_{\|} \frac{\partial^{2}}{\partial \mu \partial v_{\|}}+\frac{1}{2} v_{\|}^{2} \frac{\partial^{2}}{\partial v_{\|}^{2}}\right] \delta F_{a} .
\end{aligned}
$$

We are now left with evaluating the gyroaverage of the field-particle-like contribution $\left\langle\mathcal{C}_{M}^{F}\right\rangle$ of the equilibrium operator. At first sight, these terms have the intractable property of having a time-dependent function $S_{1}^{\prime}\left(\boldsymbol{Z}^{\prime}, t\right)$ inside the primed coordinate integral. However, the time dependence of $S_{1}^{\prime}$ is due to the electric potential $\phi$, which is a function of particle position $\boldsymbol{x}$ only. It is therefore possible to pull the time dependence outside of the primed coordinate integrals. We note that the zeroth-order 
relative velocity $u_{0}$ is periodic in the relative gyroangle $\theta^{\prime}-\theta$. Therefore, the relative velocity $u_{0}$ can be decomposed [7] into a Fourier series $u_{0}=\sum_{n=-\infty}^{\infty} u_{n} e^{i n\left(\theta^{\prime}-\theta\right)}$. Using the Fourier series decomposition and the Bessel integral representation $J_{n}(x)=$ $\frac{i^{-n}}{2 \pi} \int_{0}^{2 \pi} d \theta e^{i x \cos \theta+i n \theta}$, the $\theta^{\prime}$ integrals in the field-particle-like Rosenbluth potentials $g^{\phi_{b}}$ and $h^{\phi_{b}}$, Eq. (12), can be evaluated,

$$
\left(\begin{array}{c}
h^{\phi_{b}} \\
g^{\phi_{b}}
\end{array}\right)=\sum_{n=-\infty}^{\infty} \int d^{3} k e^{i \boldsymbol{k} \cdot \boldsymbol{x}-i n \theta} i^{n} q_{b} \phi_{k}\left(\begin{array}{c}
h_{n k} \\
g_{n k}
\end{array}\right),
$$

where

$$
\left(\begin{array}{c}
h_{n k} \\
g_{n k}
\end{array}\right)=-\frac{\Gamma_{a b}}{8 \pi} \int d \mu^{\prime} d v_{\|}^{\prime} 2 \pi F_{M b}\left[\left[\delta_{n 0}-J_{0}\left(k_{\perp} \rho_{0}^{\prime}\right) J_{n}\left(-k_{\perp} \rho_{0}^{\prime}\right)\right] \frac{\partial}{\partial \mu^{\prime}}-\sum_{l=1}^{\infty} \frac{n}{2 l} k_{\perp} \frac{\partial \rho_{0}^{\prime}}{\partial \mu^{\prime}}\left[J_{n+l}\left(-k_{\perp} \rho_{0}^{\prime}\right)-J_{n-l}\left(-k_{\perp} \rho_{0}^{\prime}\right)\right]\right]\left(\begin{array}{c}
2 u_{n}^{-1} \\
u_{n}
\end{array}\right)
$$

and $\delta_{n m}$ denotes the Kronecker delta. Note that the integrals in Eq. (17) are time independent, and can be precomputed and reused for all time steps in numerical simulations.

The $\theta^{\prime}$ integrals in the field-particle operator Rosenbluth potentials are treated in a similar way:

$$
\left(\begin{array}{c}
H_{b}\left[\delta F_{b}\right] \\
G_{b}\left[\delta F_{b}\right]
\end{array}\right)=\sum_{n=-\infty}^{\infty} \int d^{3} k e^{i \boldsymbol{k} \cdot \boldsymbol{x}-i n \theta} i^{n}\left(\begin{array}{c}
h_{n k}^{\delta F_{b}} \\
g_{n k}^{\delta F_{b}}
\end{array}\right)
$$

where

$$
\left(\begin{array}{c}
h_{n k}^{\delta F_{b}} \\
g_{n k}^{\delta F_{b}}
\end{array}\right)=-\frac{\Gamma_{a b}}{8 \pi} \int d \mu^{\prime} d v_{\|}^{\prime} 2 \pi B J_{n}\left(-k_{\perp} \rho_{0}^{\prime}\right) \delta F_{b k}\left(\begin{array}{c}
2 u_{n}^{-1} \\
u_{n}
\end{array}\right)
$$

and $\delta F_{b k}$ is the Fourier transform of $\delta F_{b}$. The integrals in the field-particle operator are time dependent. But since the gyroangle integral has been calculated, finite Larmor radius effects can be precomputed numerically, hence reducing the dimensionality of the integral from three to two.

The gyroaveraged field-particle operator $\left\langle\mathcal{C}\left[F_{M a},\left\langle\delta F_{b}\right\rangle\right]\right\rangle$ and the gyroaveraged field-particle-like part of the equilibrium collision operator $\mathcal{C}_{M}^{F}$ are combined and are given as

$$
\begin{aligned}
\left\langle\mathcal{C}_{M}^{F}\right\rangle+\left\langle\mathcal{C}\left[F_{M a}, \delta F_{b}\right]\right\rangle= & \int d^{3} k e^{i \boldsymbol{k} \cdot \boldsymbol{X}}\left\{\Gamma_{a b} \frac{m_{a}}{m_{b}} F_{M a}\left\langle\delta F_{b}\right\rangle_{k} J_{0}\left(k_{\perp} \rho_{0}\left[1-\frac{q_{a} m_{b}}{q_{b} m_{a}}\right]\right)\right. \\
& +\sum_{n=-\infty}^{\infty} F_{M a} J_{-n}\left(k_{\perp} \rho_{0}\right)\left[\left(1-\frac{m_{a}}{m_{b}}\right)\left(2 \mu \frac{\partial}{\partial \mu}+v_{\|} \frac{\partial}{\partial v_{\|}}\right) \frac{h_{n k}^{\left\langle\delta F_{b}\right\rangle}-q_{b} \phi_{k} h_{n k}}{m_{a} T_{a}}+\left(\frac{n^{2} T_{a}}{4 \mu B}+\frac{2 \mu B-T_{a}}{2 B} \frac{\partial}{\partial \mu}\right.\right. \\
& \left.\left.\left.+\frac{\mu B\left(2 \mu B-T_{a}\right)}{B^{2}} \frac{\partial^{2}}{\partial \mu^{2}}+\frac{2 \mu B v_{\|} T_{a}}{B} \frac{\partial^{2}}{\partial \mu \partial v_{\|}}+\frac{m_{a} v_{\|}^{2}-T_{a}}{2 m_{a}} \frac{\partial^{2}}{\partial v_{\|}^{2}}\right) \frac{-2\left(g_{n k}^{\delta F_{b}}-q_{b} \phi_{k} g_{n k}\right)}{T_{a}^{2}}\right]\right\} .
\end{aligned}
$$

In summary, the gyroaveraged gyrokinetic linearized Landau collision operator valid for arbitrary temperatures and masses, including the previously neglected equilibrium operator $\mathcal{C}\left[F_{M a}, F_{M b}\right]$, becomes

$$
\begin{aligned}
\left\langle\mathcal{C}\left[\left\langle F_{a}\right\rangle,\left\langle F_{b}\right\rangle\right]\right\rangle= & \left\langle\mathcal{C}\left[\delta F_{a}, F_{M b}\right]\right\rangle+\left\langle\mathcal{C}\left[F_{M a}, \delta F_{b}\right]\right\rangle \\
& +\left\langle\mathcal{C}_{M}^{T}\right\rangle+\left\langle\mathcal{C}_{M}^{F}\right\rangle
\end{aligned}
$$

where the gyroaveraged test-particle operator $\left\langle\mathcal{C}\left[\delta F_{a k}, F_{M b}\right]\right\rangle$ and the test-particle-like part $\left\langle\mathcal{C}_{M}^{T}\right\rangle$ of $\mathcal{C}\left[F_{M a}, F_{M b}\right]$ are given in Eqs. (15) and (14), respectively. The field-particle operator $\left\langle\mathcal{C}\left[F_{M a}, \delta F_{b}\right]\right\rangle$ and the field-particle-like part $\left\langle\mathcal{C}_{M}^{F}\right\rangle$ of the equilibrium operator are defined in Eq. (20). Finite Larmor radius effects are accounted for in all parts, and can be precomputed and reused in subsequent time steps when implemented in numerical simulations.

The first-order terms in $\left\langle\mathcal{C}_{M}^{T}\right\rangle$ are responsible for collisional energy exchange [10]. The energy exchange rate between species $a$ and $b$ is proportional [11] to $\left(T_{b}-T_{a}\right) v_{a b} m_{a} /\left(m_{a}+\right.$ $m_{b}$ ), and is therefore mainly important in multiple ionspecies plasmas. The magnitude of the electron-tritium energy exchange rate is smaller than the deuterium-tritium rate by a factor $\sqrt{m_{e} / m_{T}}$.

According to the gyrokinetic ordering the second-order terms in $\left\langle\mathcal{C}_{M}^{T}\right\rangle$ and $\left\langle\mathcal{C}_{M}^{F}\right\rangle$, all of which depend on the electric potential $\phi$, are of the same order as $\left\langle\mathcal{C}\left[\delta F_{a}, F_{M b}\right]\right\rangle$ and $\left\langle\mathcal{C}\left[F_{M a}, \delta F_{b}\right]\right\rangle$. The second-order terms in $\left\langle\mathcal{C}_{M}^{T}\right\rangle$ and $\left\langle\mathcal{C}_{M}^{F}\right\rangle$ can be described as "polarization-density" test- and field-particles colliding with Maxwellian particles. The "polarizationdensity" test- and field-particle collisions appear in the gyrokinetic collision operator because collisions take place between particles and not gyrocenters. The polarizationdensity particles represent deviations described by $\rho_{1}$ of particle positions from the unperturbed particle position due to electric fluctuations. This interpretation is analogous to the polarization density [1] entering the gyrokinetic Gauss's law, which describes how the charge density given a gyrocenter density function is altered due to electric fluctuations. To 
lowest order in the long wavelength limit (LWL) $k_{\perp}^{3} \rho_{0}^{3} \sim \epsilon$ [which corresponds to $n=0$ and $n=l=1$ in Eq. (17)], these terms are proportional to the magnetic field aligned component of the $\boldsymbol{E} \times \boldsymbol{B}$ vorticity $\hat{\boldsymbol{b}} \cdot \nabla \times \boldsymbol{u}_{E}$, and hence describe the effect of collisions on the particle motion associated with the electric field. The test- and field-particle operators describe drag and diffusion in gyrocenter velocity $\left(v_{\|}, \mu\right)$ space, and gyrocenter spatial diffusion perpendicular to the magnetic field. But the test- and field-particle operators also describe drag and diffusion of $\boldsymbol{E} \times \boldsymbol{B}$ and diamagnetic vorticity, which can be illustrated by taking moments of the gyrokinetic distribution function $F$. The zeroth gyrofluid [22] moment $N_{a}=\int d \mu d v_{\|} d \theta B \delta F_{a} \delta(\boldsymbol{X}-\boldsymbol{r})$ in a quasineutral simple plasma can, in the LWL, be expressed in terms of standard particle fluid moments as $N_{i} \simeq n-\nabla_{\perp}^{2}\left(\frac{\rho_{i}^{2} n}{2}\right)-\frac{n_{0}}{\Omega_{i}} \frac{\nabla_{\perp}^{2} \phi}{B}$, e.g., spatial diffusion of $\delta F_{a}$ in $\left\langle\mathcal{C}\left[\delta F_{a}, F_{M b}\right]\right\rangle$, among other effects, describes viscous damping of $\boldsymbol{E} \times \boldsymbol{B}$ vorticity. A correct description of collisional effects on, e.g., momentum and energy associated with $\boldsymbol{E} \times \boldsymbol{B}$ drift motion, therefore requires the full gyrokinetic collision operator, including the equilibrium part which was previously neglected. The equilibrium operator must also be retained for like-particle collisions. However, for like-particle collisions the equilibrium operator (9) can be evaluated using the test-particle and field-particle operators.

We would like to thank M. Salewski and J. J. Rasmussen for helpful suggestions and careful reading of the manuscript. This work was supported by an EFDA fusion researcher fellowship (WP11-FRF-RISOE/MADSEN).
[1] A. J. Brizard and T. S. Hahm, Rev. Mod. Phys. 79, 421 (2007).

[2] P. J. Catto and K. T. Tsang, Phys. Fluids 20, 396 (1977).

[3] X. Q. Xu and M. N. Rosenbluth, Phys. Fluids B 3, 627 (1991).

[4] A. J. Brizard, Phys. Plasmas 11, 4429 (2004).

[5] I. G. Abel, M. Barnes, S. C. Cowley, W. Dorland, and A. A. Schekochihin, Phys. Plasmas 15, 122509 (2008).

[6] H. Sugama, T.-H. Watanabe, and M. Nunami, Phys. Plasmas 16, 112503 (2009).

[7] B. Li and D. R. Ernst, Phys. Rev. Lett. 106, 195002 (2011).

[8] L. D. Landau, Phys. Z. Sowjetunion 10, 154 (1936).

[9] R. Balescu, Transport Processes in Plasmas, Vol. 1 (NorthHolland, Amsterdam, 1988).

[10] R. Balescu, Transport Processes in Plasmas, Vol. 2 (NorthHolland, Amsterdam, 1988).

[11] F. L. Hinton, in Basic Plasma Physics: Selected Chapters, Handbook of Plasma Physics, Vol. 1 (North-Holland, Amsterdam, 1983), p. 147.

[12] M. N. Rosenbluth, W. M. MacDonald, and D. L. Judd, Phys. Rev. 107, 1 (1957).

[13] J. Madsen, Phys. Plasmas 17, 082107 (2010).
[14] D. H. E. Dubin, J. A. Krommes, C. Obermann, and W. W. Lee, Phys. Fluids 26, 3524 (1983).

[15] W. W. Lee, Phys. Fluids 26, 556 (1983).

[16] A. G. Peeters, Y. Camenen, F. J. Casson, W. A. Hornsby, A. P. Snodin, D. Strintzi, and G. Szepesi, Comput. Phys. Commun. 180, 2650 (2009).

[17] T. Vernay, S. Brunner, L. Villard, B. F. McMillan, S. Jolliet, T. M. Tran, A. Bottino, and J. P. Graves, Phys. Plasmas 17, 122301 (2010).

[18] B. D. Scott, A. Kendl, and T. Ribeiro, Contrib. Plasma Phys. 50, 228 (2010).

[19] A. Bottino, T. Vernay, B. Scott, S. Brunner, R. Hatzky, S. Jolliet, B. F. McMillan, T. M. Tran, and L. Villard, Plasma Phys. Controlled Fusion 53, 124027 (2011).

[20] T. Görler, X. Lapillonne, S. Brunner, T. Dannert, F. Jenko, F. Merz, and D. Told, J. Comput. Phys. 230, 7053 (2011).

[21] P. Helander and D. J. Sigmar, Collisional Transport in Magnetized Plasmas (Cambridge University Press, Cambridge, UK, 2005).

[22] J. Madsen, O. E. Garcia, J. S. Larsen, V. Naulin, A. H. Nielsen, and J. J. Rasmussen, Phys. Plasmas 18, 112504 (2011). 